\title{
The effect of hemodynamically-guided hypotensive therapy in one-year observation: Randomized, prospective and controlled trial (FINEPATH study)
}

\author{
Paweł Krzesiński, Grzegorz Gielerak, Adam Stańczyk, Katarzyna Piotrowicz, \\ Beata Uziębło-Życzkowska, Małgorzata Banak, Małgorzata Kurpaska, \\ Łukasz Michalczyk, Agnieszka Jurek, Kalina Wolszczak, Agata Galas, \\ Agnieszka Wójcik, Andrzej Skrobowski
}

Department of Cardiology and Internal Diseases, Military Institute of Medicine, Warsaw, Poland

\begin{abstract}
Background: The use of impedance cardiography (ICG) revealed to provide beneficial blood pressure (BP) lowering effect. However, the follow-up in previous trials was short and brachial $B P$ was the only evaluated hemodynamic variable. Thus, we aimed to estimate the influence of ICG-guided therapy on brachial and central BP, impedance-derived hemodynamic profile and echocardiographic features after 12 months in a randomized, prospective and controlled trial (NCT01996085).

Methods: One hundred and forty-four hypertensives were randomly assigned to groups of empiric (GE) and ICG-guided therapy (HD). Office BP, ambulatory BP monitoring, central $B P$ and echocardiography (left ventricular hypertrophy and diastolic function assessment) were performed before and after 12 months of treatment.

Results: Blood pressure reduction was higher in HD (office BP: 21.8/14.1 vs. 19.9/11.8 $\mathrm{mm} \mathrm{Hg;}$ mean 24-h BP: 19.0/10.9 vs. $14.4 / 9.2 \mathrm{~mm} \mathrm{Hg}$ ). However, the only statistically significant differences were: percentage of patients achieving BP reduction of minimum $20 \mathrm{~mm} \mathrm{Hg}$ for office diastolic BP (27.3\% vs. $12.1 \%$; $p=0.034)$ and mean 24 - $h$ systolic BP $(49.1 \%$ vs. $27.3 \%$; $p=0.013$ ). More pronounced improvement in the left ventricular diastolic dysfunction (delta $E / A 0.34$ vs. $0.12, p=0.017$ ) was the only other beneficial hemodynamic effect.

Conclusions: Beneficial BP lowering effect of hemodynamically-guided pharmacotherapy, observed previously in short-term observation, persists over time. Hemodynamic effects of such a treatment approach, especially those of prognostic value (central BP, myocardial hypertrophy), should be evaluated in further studies including patients with resistant hypertension, heart failure, diabetes mellitus and chronic kidney disease. (Cardiol J 2016; 23, 2: 132-140)
\end{abstract}

Key words: hypertension, blood pressure, impedance cardiography, cardiovascular diseases, antihypertensive agents, hemodynamics

Address for correspondence: Paweł Krzesiński, PhD, Department of Cardiology and Internal Diseases, Military Institute of Medicine, ul. Szaserów 128, 04-141 Warszawa 44, Poland, tel: +48 2218163 89, fax: +48 228108089 , e-mail: pkrzesinski@wim.mil.pl

Received: 02.11.2015 Accepted: 06.01.2016 


\section{Introduction}

Arterial hypertension ( $\mathrm{AH})$ is the main widespread cardiovascular risk factor in the world's population. Unfortunately, the majority of hypertensives still do not achieve satisfactory blood pressure (BP) control [1,2]. Current guidelines $[2,3]$ emphasize a need for personalized hypotensive therapy. It may be supported by individualized assessment of hemodynamic alterations associated with increased BP. One of the promising diagnostic techniques is impedance cardiography (ICG), useful in the identification of hyperdynamic heart function, fluid retention, and increased vascular resistance. Hemodynamically-guided pharmacotherapy based on impedance variables, such as cardiac index (CI), thoracic fluid content (TFC) and systemic vascular resistance index (SVRI), was previously evaluated in randomized, prospective and controlled trials and revealed to be more beneficial than the empiric treatment [4-8]. However, the period of observation in those trials was short (3 months) and BP reduction was the only evaluated hemodynamic effect.

Thus, there is no evidence (1) if this effect persists in longer observation period and (2) whether this therapeutic approach has influence on other features related to cardiovascular hemodynamics, especially these of prognostic value (central BP, myocardial hypertrophy) [2, 9].

Therefore, the aim of this study was to estimate the influence of ICG-guided antihypertensive therapy on: (1) brachial and (2) central BP, (3) impedance-derived hemodynamic profile and (4) echocardiographic indices of left chambers' morphology and function evaluated after 12 months in a randomized, prospective and controlled trial.

\section{Methods}

\section{Study population}

The study group consisted of patients with at least 3-month history of AH defined according to the European Society of Cardiology (ESC) guidelines [2]. Exclusion criteria were: (1) confirmed secondary $\mathrm{AH},(2) \mathrm{AH}$ treated with three or more medicines before recruitment, (3) significant heart failure (left ventricular ejection fraction $<45 \%$ ), (4) cardiomyopathy (i.e. hypertrophic cardiomyopathy), (5) significant heart rhythm disorders, (6) significant valvular disease, (7) kidney failure (estimated glomerular filtration rate $<60 \mathrm{~mL}$ / $/ \mathrm{min} / 1.73 \mathrm{~m}^{2}$ ), (8) chronic obstructive pulmonary disease, (9) diabetes, (10) polyneuropathy, (11) peripheral vascular disease, (12) age $<18$ years.

Study group comprised of patients recruited for prospective, randomized and controlled study, performed in the Department of Cardiology and Internal Diseases of Military Institute of Medicine (ClinicalTrials.gov NCT01996085) and conducted according to the Good Clinical Practice guidelines and the Declaration of Helsinki, with the approvals of the local ethics committee. Each patient provided written informed consent to participate in the study.

\section{Study design}

The study was randomized (1:1), prospective and simultaneously controlled by conventional treatment. Initial clinical evaluation was performed via face to face or telephone conversations. Patients who had taken medicines before the study were advised to discontinue using them (minimum 7 days of pharmacological "wash-out"). Next, all patients underwent complete clinical examination including: interview and physical examination, office blood pressure measurement (OBPM), ambulatory blood pressure monitoring (ABPM), impedance cardiography, applanation tonometry, and echocardiography.

The subjects were allocated to two groups according to the pre-established random order (by using the method of randomly permuted blocks available at www.randomization.com): (1) empiric (GE) and (2) hemodynamic (HD). Treatment choice in both groups was made by independent researchers.

The evaluation of treatment effects was performed after 12 months and blinded to the group allocation. Differences between groups in BP reduction and obtained BP control were considered main final points (per protocol analysis). Figure 1 shows the protocol flowchart for observation time of mean $376 \pm 25$ days. The subjects were evaluated 3 times (no additional interim visits were performed). Twenty-three patients were excluded from the final analysis (GE: 7 subjects; HD: 16 subjects). Twenty-two of them resigned from control visit and 1 woman stopped the pharmacotherapy due to pregnancy. The final analysis comprised of 121 subjects (Fig. 1).

\section{Office blood pressure measurement \\ Office systolic and diastolic blood pressure measurement was performed during morning hours (7.30 a.m.-8.30 a.m.) automatically (Omron M4 Plus, Japan) by a technique compliant with the ESC guidelines [2].}




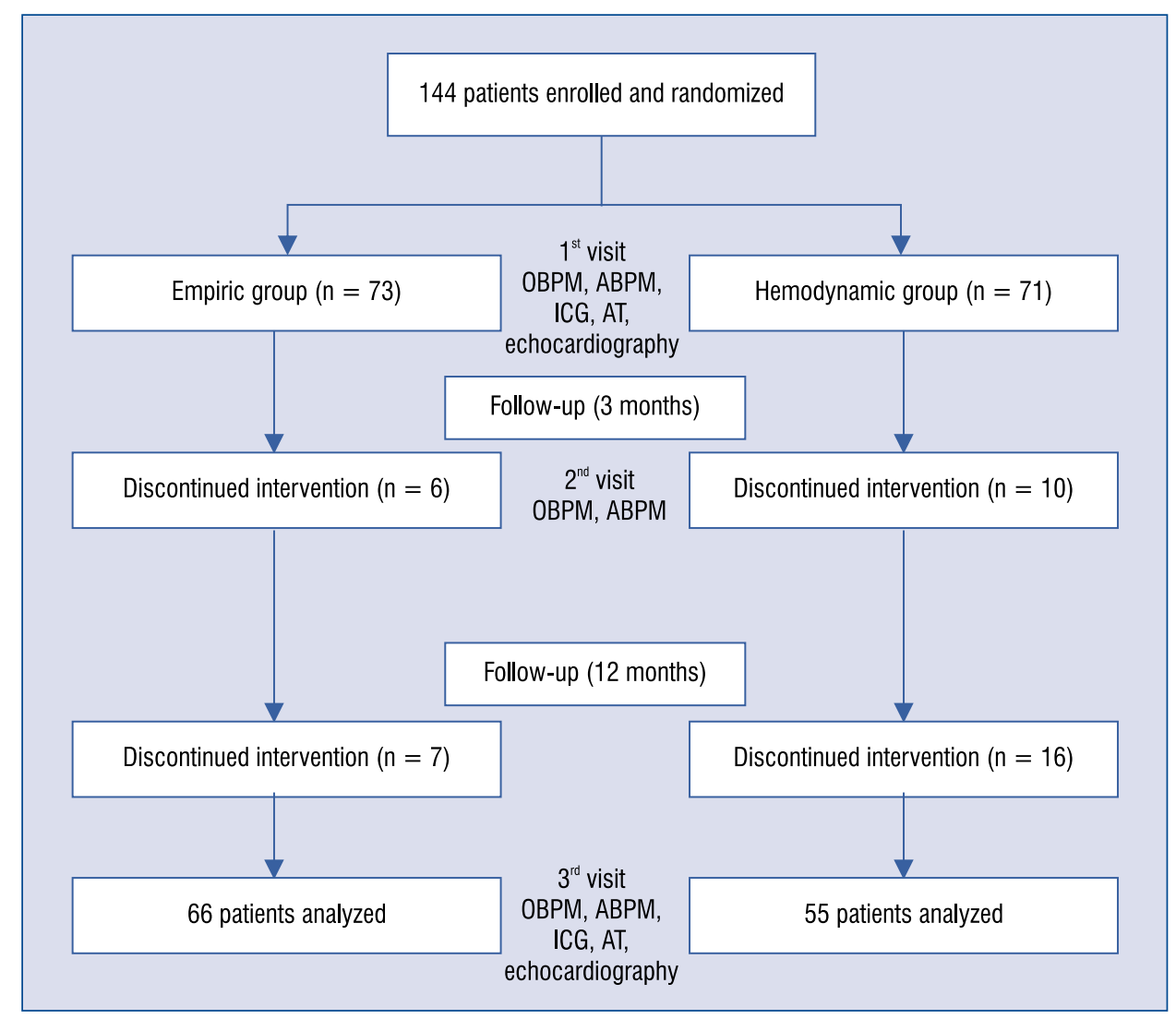

Figure 1. The flowchart of patients (numbers of discontinued interventions after 12 months are cumulative); ABPM - ambulatory blood pressure monitoring; AT — applanation tonometry; ICG — impedance cardiography; OBPM - office blood pressure measurement.

\section{Ambulatory blood pressure monitoring}

Ambulatory blood pressure monitoring started in the morning hours (Spacelabs 90207, Spacelabs, Medical Inc., Redmond, USA). Time from 6 a.m. to 10 p.m. was considered daily activity period (daytime) with automatic BP measurements in 10-min intervals. During night rest (10 p.m. -6 a.m.) the measurements were performed every $30 \mathrm{~min}$. $\mathrm{Pa}-$ tients were recommended to adjust their circadian activity to those periods of time. BP thresholds used to define $\mathrm{AH}$ were set according to the ESC guidelines [2].

\section{Applanation tonometry}

The assessment of central BP and augmentation index (AI) was performed noninvasively using the SphygmoCor ${ }^{\circledR}$ system (AtCor Medical Inc Pty Ltd, Sydney, Australia). Radial artery pressure waveforms were recorded at left wrist using applanation tonometry with a high-fidelity micromanometer (Millar Instruments, Houston, Texas) and processed by SphygmoCor ${ }^{\circledR}$ software (version 9.0; AtCor Medical Inc Pty Ltd., Sydney, Australia). Corresponding aortic pressure waveform was generated using a validated transfer function [10]. As a result, central systolic blood pressure (CSBP $[\mathrm{mm} \mathrm{Hg}]$ ), central diastolic blood pressure (CDBP $[\mathrm{mm} \mathrm{Hg}])$ and central pulse pressure $(\mathrm{CPP}[\mathrm{mm} \mathrm{Hg}])$ were derived. Augmentation pressure (AP [mm $\mathrm{Hg}]$ ) was calculated as the maximum systolic pressure minus pressure at the inflection point and AI ([\%]) as AP $\times 100 / \mathrm{CPP}$.

\section{Echocardiography}

Two-dimensional echocardiography was performed using standard parasternal, apical, and subcostal views (2.5 MHz transducer; VIVID S6 GE Medical System, Wauwatosa, WI, USA). The dimensions of left atrium (LA), left ventricular end diastolic diameter (LVEDD) and interventricular septum diameter were measured in the parasternal long-axis view in the late diastole of the LA and left ventricle (LV), respectively. Left ventricular ejection fraction was calculated according to the Simpson's formula. The left ventricular hypertrophy (LVH) was diagnosed according to the formula recommended by the American Society of Echocardiography for estimation of the left ventricular 
mass index (LVMI), indexed to body surface area (cutoff values for men LVMI $>115 \mathrm{~g} / \mathrm{m}^{2}$, for women $>95 \mathrm{~g} / \mathrm{m}^{2}$ ).

Mitral valve inflow was recorded in the apical 4-chamber view with pulsed wave Doppler gate positioned in $\mathrm{LV}$ at the level of mitral valve edges. The following parameters were measured: mitral inflow early (E) and late (A) phase ratio (E/A) and phase $\mathrm{E}$ deceleration time. Tissue Doppler imaging was performed in the apical views to acquire mitral annular velocity, mitral septal annulus early diastolic velocity (e') was measured and based on this the E/e' ratio was calculated. Diagnosis of the LV diastolic dysfunction was based on the current guidelines [11]. The study group comprised only of patients with normal and mildly impaired LV diastolic function (impaired relaxation diastolic filling pattern).

\section{Impedance cardiography}

All ICG measurements were performed using the Niccomo $^{\mathrm{TM}}$ device (Medis, Ilmenau, Germany) after $10 \mathrm{~min}$ of rest in a supine position. Blood pressure measurement was performed automatically every 2 min with an arm cuff. Other hemodynamic parameters were measured with a beat-to-beat method. The TFC, CI, SVRI, heart rate (HR) values measured in the $5^{\text {th }}$ min of the examination were taken into account in the treatment algorithm. The cutoff values for TFC, SVRI, CI and HR (from the $5^{\text {th }}$ min of ICG examination) defined hemodynamic profile: (1) hyperconstrictive: in case of SVRI $>2500 \mathrm{dyn} / \mathrm{s} / \mathrm{m}^{2} / \mathrm{cm}^{5}$, (2) hyperdynamic: $\mathrm{CI}>4.2$ $1 / \mathrm{min} / \mathrm{m}^{2}$ and/or HR $>80 / \mathrm{min}$, (3) hypervolemic profile: $\mathrm{TFC}>341 / \mathrm{kOhm}$ for men and $>24$ $1 / \mathrm{kOhm}$ for women, (4) balanced profile - hemodynamic parameters below established threshold values. The subjects requiring combined therapy with regard to significantly increased $\mathrm{BP}$ (average office $\mathrm{BP}>160 / 100 \mathrm{~mm} \mathrm{Hg}$ and/or average 24-h $\mathrm{BP}>140 / 90 \mathrm{~mm} \mathrm{Hg}$ ) were also distinguished.

\section{Treatment}

Non-pharmacological treatment was administered according to the current ESC guidelines [2]. Pharmacotherapy included: lisinopril (angiotensin converting enzyme inhibitor [ACEI]), telmisartan (angiotensin receptor blocker [ARB]), hydrochlorothiazide/indapamide (diuretic), metoprolol/ /nebivolol (beta-blocker [BB]), amlodipine (calcium blocker [CB]).

The arbitrarily predetermined treatment algorithm in HD group was based on our own data collected in the cohort of hypertensive patients and the analysis of the previous reports $[5,6,12-$ $-15]$. Cutoffs for CI and SVRI were adopted from the previous studies of Taler et al. [5] and Smith et al. [6]. Increased rest HR ( $>80 / \mathrm{min}$ ) was considered an indication for $\mathrm{BB}$ use due to the fact that it had been reported to be unfavorable for prognosis [15]. The different cut-off values for TFC for men and women were defined based on sex-dependant computational calculation used in Niccomo device.

The first step of drug choice was based on the hemodynamic profile (hyperdynamic profile - BB, hypervolemic - diuretic, hyperconstrictive vasodilator/s). Combined therapy was applied in cases of complex hemodynamic disturbances and to patients demanding combined polytherapy (BP criteria mentioned above). This algorithm was described in detail in our previous study [7]. Patients in the GE group were treated according to the current ESC guidelines [2]. With most patients the first drug choice was ACEI and $\mathrm{CB}$, the most preferred combinations were: ACEI with diuretic and ACEI with CB.

\section{Statistical analysis}

On the basis of the results of the previous studies [5-7], the sample size for minimum change in $\mathrm{BP}$ reduction of $6 \mathrm{~mm} \mathrm{Hg}$ after 12 months of follow-up was calculated as 45 patients per treatment group (a-error 5\%, statistical power $80 \%$ ). The statistical analysis was performed using Statistica 7.0 (StatSoft, Inc., Tulsa, USA). The distribution and normality of the data were assessed by visual inspection and the Kolmogorov-Smirnov test. Continuous variables were presented as means \pm standard deviation. Treatment effects were compared with the use of ANOVA/Mann-Whitney U-test for continuous variables (change in BP) and $\chi^{2}$ test/Fisher's exact test for categorical variables (percentage of achieved reduction in $\mathrm{BP}$ of minimum $10 \mathrm{~mm} \mathrm{Hg} / 20 \mathrm{~mm} \mathrm{Hg}$ ). The change of chosen variables was calculated as [delta $]=$ [absolute value after 12 months $]-[$ baseline absolute value]. $\mathrm{P}<0.05$ was considered statistically significant.

\section{Results}

\section{Baseline clinical data}

The final analysis involved 121 patients ( 83 men) of average age $46.4 \pm 10.1$ years (spread from 20 to 68 years). Most of them (81.0\%) were mildly hypertensive (grade 1) and only $19.0 \%$ of them presented AH grade 2 or $3.20 .7 \%$ of them were previously treated with hypotensive drugs. 
Table 1. Basic characteristics; data presented as mean \pm standard deviation and numbers (percentages); no statistically significant differences between empiric (GE) group and hemodynamic group (HD) were noted.

\begin{tabular}{lcc}
\hline Variable & \multicolumn{2}{c}{ Whole group $(\mathbf{n}=\mathbf{1 2 1})$} \\
\cline { 2 - 3 } & GE $(\mathbf{n}=\mathbf{6 6})$ & HD $(\mathbf{n}=\mathbf{5 5})$ \\
\hline Men & $47(71.2 \%)$ & $36(65.5 \%)$ \\
Age [years] & $46.1 \pm 9.8$ & $46.7 \pm 10.5$ \\
Body mass index [kg/m²] & $28.7 \pm 4.1$ & $29.2 \pm 4.0$ \\
Office SBP [mm Hg] & $140.9 \pm 12.9$ & $142.0 \pm 14.3$ \\
Office DBP [mm Hg] & $90.0 \pm 9.5$ & $90.8 \pm 9.6$ \\
Mean 24-h SBP [mm Hg] & $139.5 \pm 12.0$ & $142.1 \pm 11.9$ \\
Mean 24-h DBP [mm Hg] & $88.2 \pm 7.8$ & $88.3 \pm 11.9$ \\
Left ventricular hypertrophy & $9(13.6 \%)$ & $4(7.3 \%)$ \\
Left ventricular diastolic dysfunction & $20(30.3 \%)$ & $12(21.8 \%)$ \\
\hline
\end{tabular}

DBP — diastolic blood pressure; SBP — systolic blood pressure

Table 2. Blood pressure values within subsequent visits; data presented as mean \pm standard deviation, no statistically significant differences between empiric (GE) group and hemodynamic group (HD) for respective variables.

\begin{tabular}{|c|c|c|c|c|c|c|c|c|}
\hline \multirow[t]{2}{*}{ Variable } & \multicolumn{4}{|c|}{ GE $(n=66)$} & \multicolumn{4}{|c|}{$H D(n=55)$} \\
\hline & $1^{\text {st }}$ visit & $2^{\text {nd }}$ visit & $3^{\text {rd }}$ visit & $\mathbf{P}$ & $1^{\text {st }}$ visit & $2^{\text {nd }}$ visit & $3^{\text {rd }}$ visit & $\mathbf{P}$ \\
\hline $\begin{array}{l}\text { Office SBP } \\
\text { [mm Hg] }\end{array}$ & $140.9 \pm 12.9$ & $124.1 \pm 11.8$ & $120.9 \pm 10.8$ & $<0.00001$ & $142.0 \pm 14.3$ & $123.5 \pm 9.9$ & $120.2 \pm 8.1$ & $<0.00001$ \\
\hline $\begin{array}{l}\text { Office DBP } \\
{[\mathrm{mm} \mathrm{Hg]}}\end{array}$ & $90.0 \pm 9.5$ & $81.0 \pm 10.0$ & $78.2 \pm 6.4$ & $<0.00001$ & $90.8 \pm 9.6$ & $79.4 \pm 6.7$ & $76.7 \pm 6.4$ & $<0.00001$ \\
\hline $\begin{array}{l}\text { Mean 24-h } \\
\text { SBP [mm Hg] }\end{array}$ & $139.5 \pm 12.0$ & $126.1 \pm 11.5$ & $125.0 \pm 9.9$ & $<0.00001$ & $142.1 \pm 11.9$ & $124.9 \pm 11.5$ & $123.1 \pm 8.9$ & $<0.00001$ \\
\hline $\begin{array}{l}\text { Mean 24-h } \\
\text { DBP [mm Hg] }\end{array}$ & $88.2 \pm 7.8$ & $78.9 \pm 8.3$ & $78.9 \pm 7.4$ & $<0.00001$ & $88.3 \pm 11.9$ & $78.2 \pm 7.8$ & $77.4 \pm 6.7$ & $<0.00001$ \\
\hline
\end{tabular}

DBP — diastolic blood pressure; SBP — systolic blood pressure

No significant differences in basic clinical characteristics between GE and HD subjects were observed (Table 1).

\section{Treatment effects - brachial blood pressure \\ Reduction of BP absolute values due to treat-} ment was observed in both groups. However, no statistically significant difference between GE and HD was noted (Table 2). The benefit from the hemodynamically-guided therapy revealed to be more evident when comparing delta systolic BP (Fig. 2) but the differences still did not reach statistical significance. The only statistically significant differences were obtained for the percentage of patients achieving BP reduction of minimum $20 \mathrm{~mm} \mathrm{Hg}$ for office diastolic BP and mean 24-h systolic BP (Fig. 3).
Treatment effects - impedance cardiography, applanation tonometry and echocardiography

The use of ICG did not significantly influence the effects of 12-month therapy on other analyzed hemodynamic and echocardiographic characteristics. There were no significant differences between GE and $\mathrm{HD}$ in central BP, basic impedance indices (HR, CI, TFC, SVRI), left chamber dimensions (LVEDD, LA), cardiac muscle mass (LVMI) and echocardiographic indices of LV diastolic function (Table 3). However, the analysis in a subgroup of patients with LV diastolic dysfunction revealed slightly a better effect of hemodynamically-guided intervention - better improvement (change) in $\mathrm{E} / \mathrm{A}$ (GE vs. HD: 0.12 vs. $0.34, \mathrm{p}=0.017)$, e' ( 2.67 vs. $2.13 \mathrm{~cm} / \mathrm{s}$, $\mathrm{p}=0.699)$ and $\mathrm{E} / \mathrm{e}^{\prime}(-0.77$ vs. $-0.82, \mathrm{p}=0.839)$. 


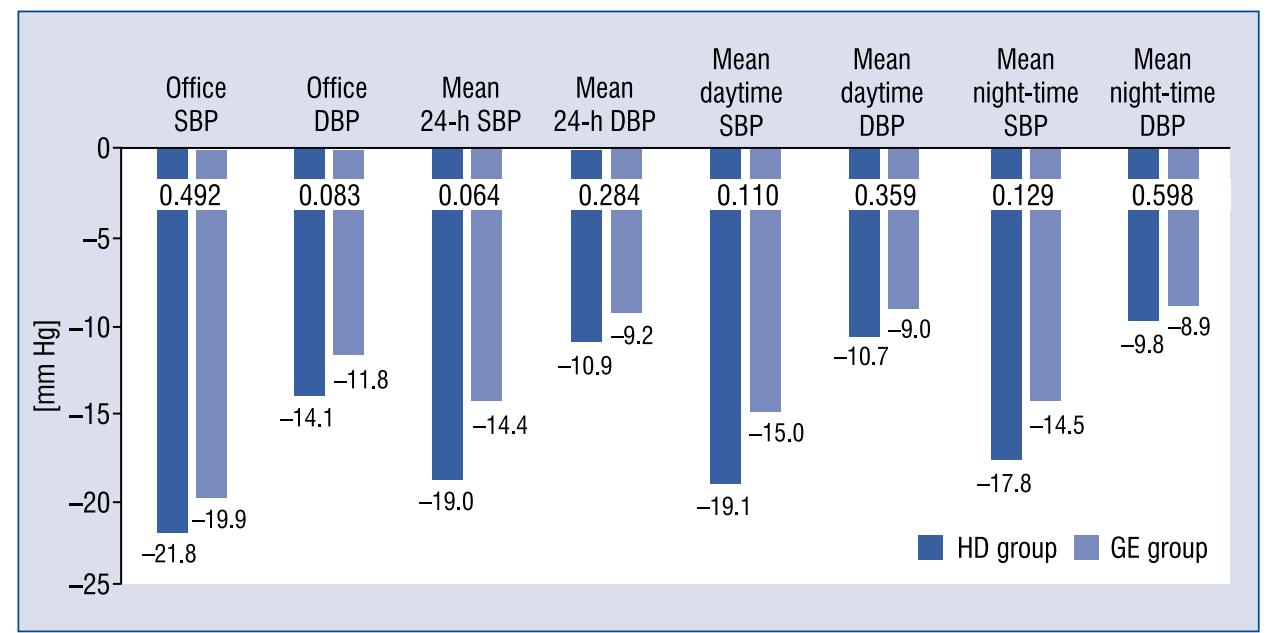

Figure 2. The comparison of the effect between empiric (GE) and hemodynamically guided treatment (HD) within BP change ( $p$ value presented on the bars); DBP — diastolic blood pressure; SBP — systolic blood pressure.

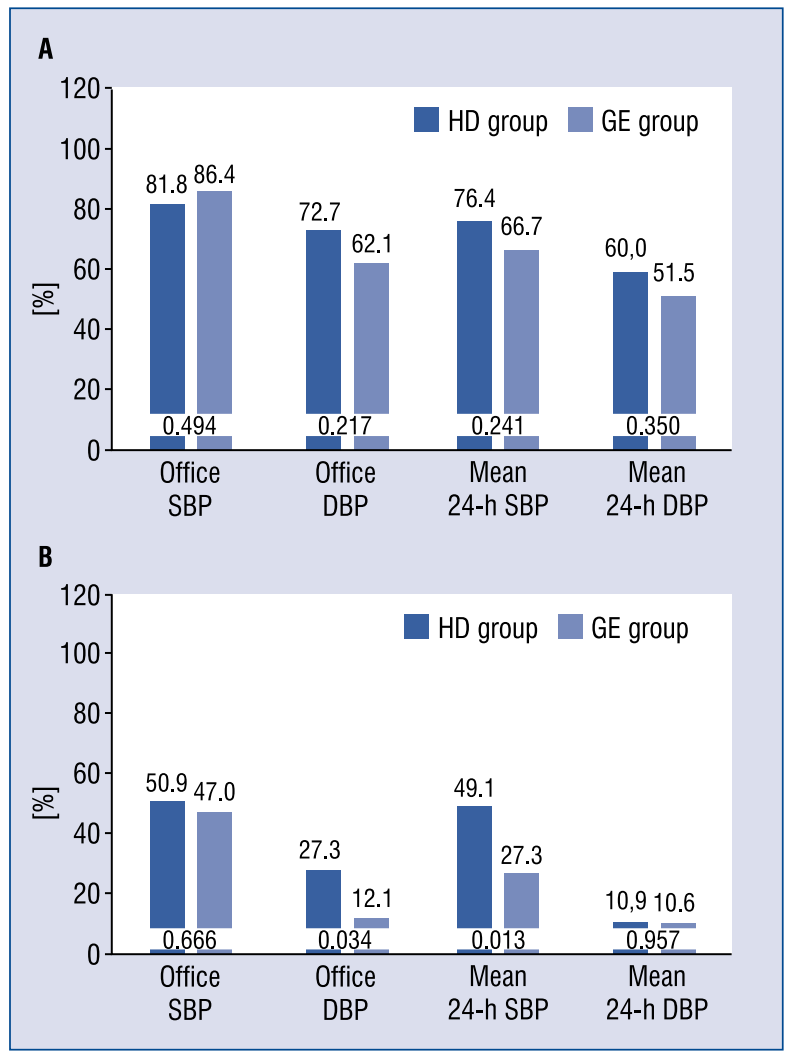

Figure 3. The comparison of the effect between empiric (GE) and hemodynamically guided treatment (HD) within achieved reduction in blood pressure of minimum $10 \mathrm{~mm} \mathrm{Hg} \mathrm{(A)} \mathrm{and} \mathrm{of} \mathrm{minimum} 20 \mathrm{~mm} \mathrm{Hg}$ (B); DBP diastolic blood pressure; SBP — systolic blood pressure.

Such an analysis was not performed in the subgroup of patients with LVH because of low sample size $(\mathrm{n}=13)$.

\section{Pharmacotherapy}

The most commonly administered drugs in both groups were renin-angiotensin-aldosterone system's blockers (ACEI/ARB) and diuretics (Table 4). The GE patients were more frequently treated with ACEI and $\mathrm{CB}$, whereas the HD patients with diuretics and $\mathrm{BB}$. However, no statistically significant difference was noted. Likewise, the percentage of patients demanding intensification of pharmacotherapy during second visit was comparable (GE vs. HD: $19.7 \%$ vs. $18.2 \%, \mathrm{p}=0.832)$.

\section{Discussion}

Blood pressure control still remains a challenge in $\mathrm{AH}$. Thus, the search for noninvasive tools useful in individualized hemodynamic assessment of hypertensives seems to be justified. We present the results of FINEPATH study which aimed at evaluating whether the beneficial effect of hemodynamically-guided therapy, previously reported in short-term observations, persisted over 1 year and whether it was visible in other clinically relevant characteristics. Our results encourage the use of ICG while choosing the antihypertensive pharmacotherapy. We observed clinically relevant better BP lowering effect and slightly more pronounced improvement of the LV diastolic function in subjects treated according to ICG-based algorithm. No significant differences were observed in left chambers morphology, LV systolic function and central BP. 
Table 3. Treatment effects assessed by impedance cardiography, applanation tonometry and echocardiography; data presented as mean \pm standard deviation and numbers (percentages).

\begin{tabular}{|c|c|c|c|}
\hline Variable & $\begin{array}{l}\text { Empiric group } \\
\qquad(n=66)\end{array}$ & $\begin{array}{l}\text { Hemodynamic group } \\
\qquad(\mathrm{n}=55)\end{array}$ & $\mathbf{P}$ \\
\hline \multicolumn{4}{|l|}{ Impedance cardiography } \\
\hline Delta HR [bpm] & $-2.4 \pm 9.6$ & $-5.5 \pm 10.6$ & 0.092 \\
\hline Delta $\mathrm{Cl}\left[\mathrm{l} / \mathrm{min} / \mathrm{m}^{2}\right]$ & $-0.06 \pm 0.59$ & $-0.11 \pm 0.70$ & 0.831 \\
\hline Delta TFC [1/kohm] & $-0.43 \pm 4.6$ & $0.79 \pm 3.57$ & 0.164 \\
\hline Delta SVRI $\left[\mathrm{dyn}{ }^{*} \mathrm{~s}^{*} \mathrm{~m}^{2} / \mathrm{cm}^{5}\right]$ & $-280.6 \pm 433.8$ & $-251.7 \pm 426.1$ & 0.873 \\
\hline \multicolumn{4}{|l|}{ Applanation tonometry } \\
\hline Delta CSBP [mm Hg] & $16.7 \pm 14.6$ & $14.2 \pm 14.0$ & 0.345 \\
\hline Delta CDBP [mm Hg] & $11.6 \pm 10.0$ & $11.2 \pm 9.0$ & 0.942 \\
\hline Delta CPP [mm Hg] & $5.1 \pm 8.4$ & $3.2 \pm 9.2$ & 0.243 \\
\hline Delta Al [\%] & $0.74 \pm 11.1$ & $0.04 \pm 10.1$ & 0.595 \\
\hline \multicolumn{4}{|l|}{ Echocardiography } \\
\hline Delta LVEDD [mm] & $0.10 \pm 1.69$ & $-0.28 \pm 3.50$ & 0.312 \\
\hline Delta LA [mm] & $0.50 \pm 2.51$ & $0.74 \pm 2.27$ & 0.592 \\
\hline Delta LVMI $\left[\mathrm{g} / \mathrm{m}^{2}\right]$ & $-0.53 \pm 15.0$ & $2.33 \pm 14.0$ & 0.579 \\
\hline Delta e' $[\mathrm{cm} / \mathrm{s}]$ & $0.80 \pm 2.67$ & $0.90 \pm 2.39$ & 0.830 \\
\hline Delta E/e' [-] & $-0.22 \pm 1.93$ & $-0.26 \pm 1.97$ & 0.682 \\
\hline Delta E/A [-] & $0.06 \pm 0.32$ & $0.12 \pm 0.33$ & 0.229 \\
\hline Delta LVEF [\%] & $1.26 \pm 4.17$ & $1.58 \pm 4.24$ & 0.656 \\
\hline
\end{tabular}

$\mathrm{Al}$ - augmentation index; $\mathrm{Cl}$ - cardiac index; $\mathrm{CDBP}$ - central diastolic blood pressure; CSBP — central systolic blood pressure; CPP — central pulse pressure; $e^{\prime}$ - mitral septal annulus early diastolic velocity; $E / A$ - mitral flow early $(E)$ and late $(A)$ phase ratio; $E / e^{\prime}$ - mitral flow early $(E)$ phase and mitral septal annulus early diastolic velocity ( $\left.e^{\prime}\right)$ ratio; HR - heart rate; LA - left atrium diameter; LVEDD - left ventricular end diastolic diameter; LVEF — left ventricular ejection fraction; SVRI — systemic vascular resistance index; TFC — thoracic fluid content

Table 4. Pharmacotherapy (data presented as numbers (percentages).

\begin{tabular}{lccc}
\hline & Empiric group $(\mathbf{n}=66)$ & Hemodynamic group $(\mathbf{n}=\mathbf{5 5})$ & $\mathbf{P}$ \\
\hline ACE inhibitor & $52(78.8)$ & $38(69.1)$ & 0.224 \\
ARB & $8(12.1)$ & $8(14.6)$ & 0.695 \\
Diuretics & $17(25.8)$ & $23(41.8)$ & 0.062 \\
Beta-blocker & $11(16.7)$ & $16(29.1)$ & 0.102 \\
Calcium blocker & $12(18.8)$ & $7(12.7)$ & 0.412 \\
\hline
\end{tabular}

ACE — angiotensin converting enzyme; ARB — angiotensin receptor blocker

\section{Blood pressure (brachial) lowering effects}

Our results suggest persistent benefit from the hemodynamically-guided antihypertensive therapy. Patients treated according to ICG achieved slightly greater BP reduction in both OBPM and ABPM. That effect was more pronounced among those with higher BP reduction (Fig. 3B). However, the statistical significance was achieved only for percentage of patients achieving $\mathrm{BP}$ reduction of minimum $20 \mathrm{~mm} \mathrm{Hg}$ for office diastolic $\mathrm{BP}$ and mean 24-h systolic BP. The uncertainty of other results seems unlikely because better BP reduction was observed independently in OBPM and
ABPM. Even those statistically non-significant differences we interpret as clinically important because a slight decrease in BP revealed to be related to lower risk of overall mortality, coronary disease and stroke [16]. We suppose that the expected benefit from the ICG-guided therapy was limited by sample size and the early stage of $\mathrm{AH}$ (see more in 'Limitations of the study' section). We would also like to emphasize that subjects in GE were treated by experienced cardiologists, of higher expertise than general practitioners, which carry the main weight of medical care over young hypertensives. It could cause underestimation of 
the expected advantage of ICG in the real world of ambulatory care.

Our results are in line with previous studies in this area evaluating the effect of ICG-guided therapy in short-term observation $[5-7,17]$. Smith et al. [6] evaluated 164 patients with essential AH and obtained better office BP control in HD group ( $77 \%$ vs. $57 \%, \mathrm{p}<0.001$ ). In another prospective, randomized and controlled trial Taler et al. [5] used ICG-based therapy in 104 resistant hypertensives (average BP 171/89 $\mathrm{mm} \mathrm{Hg}$ ) and also achieved higher frequency of goal office BP in HD group (56\% vs. $33 \%$ in GE group, $\mathrm{p}<0.05$ ). Likewise, Fadl Elmula et al. [17], in a small sample of patients with resistant $\mathrm{AH}(\mathrm{n}=19)$, reported better BP lowering effect of pharmacotherapy adjusted to the individual hemodynamic profile than renal denervation. The effect of ICG-guided therapy in aforementioned study was less impressive than ours but the characteristics of our population were also different. Lower baseline BP could significantly limit the disclosure of advantage from individualized treatment. As revealed in our previous analysis the benefit from ICG-guided therapy rose with the complexity of $\mathrm{AH}$ and was observed only in subjects with higher baseline BP [18].

\section{Out-of-brachial BP lowering effects}

We did not observe any benefit of the intervention in the analysis of change in the left chamber dimensions and LV muscle mass, as it was reported by Fagard et al. [19]. In that metaanalysis, the reduction of LVMI was clinically relevant $(11.0 \% \pm$ $\pm 0.60 \%, \mathrm{p}<0.001)$ but almost half of the patients (43.6\%) presented with baseline LVH. Moreover, the authors proved that regression of LVH was related to longer study duration. No influence of antihypertensive treatment on LVMI in our study cohort can be explained by early stage of cardiovascular dysfunction (hypertensives without any other serious diseases), very low prevalence of LVH (10.7\%) and 12-month (quite short) period of follow-up.

The comparative analysis did not reveal any intergroup difference in change of impedance parameters characterizing the hemodynamic profile. However, those results could be influenced by different, even opposite, hemodynamic action of drugs. Relatively high standard deviations of those variables suggest that the individual effects were underestimated by the use of averaging in general comparison. True hemodynamic effects could be revealed in separate analysis of subgroups (treated with different classes of drugs) which was not conducted because of small sample size.

The trend to greater HR reduction in HD could partly explain why the higher brachial BP reduction was not reflected in central $\mathrm{BP}$ change. It could be a result of more frequent use of $\mathrm{BB}$ in $\mathrm{HD}$ which was less effective in central BP lowering than vasodilators (preferred in GE) [20]. The mechanism was explained in a simple manner by Nieminen et al. [21]. Beta-blocker slows down the HR, increases the filling time of the $\mathrm{LV}$ and stroke volume. As a result, the pulsatile LV outflow and CPP increase.

\section{Pharmacotherapy}

Although some trends in the analysis of pharmacotherapy were observed (subjects in GE were more frequently treated with vasodilators and in $\mathrm{HD}$ with diuretics and $\mathrm{BB}$ ), they did not reveal significant differences between GE and HD. It suggests that in comparison to our previous study [7], empiric treatment was more intensive. It could partly explain slightly lower advantage of hemodynamically-guided intervention.

\section{Limitations of the study}

We are aware that the main limitation of the study was the sample size, which influenced the statistical power of the comparison. Basing on the previous studies [5-7] we calculated the sample size (45 per group) assuming intergroup difference (GE vs. HD) in delta BP as $6 \mathrm{~mm} \mathrm{Hg}$. Finally, the highest observed difference in our analysis was $4.6 \mathrm{~mm} \mathrm{Hg}$ (delta 24-h mean systolic BP). According to the statistical approach (power analysis), the sample size in this case should not be less than 76 per group. The fact of enrollment of relatively healthy patient cohort also mattered. We are aware that our results should be extrapolated on general population with care because we recruited hypertensives without other serious chronic diseases. On the other hand, the time of follow-up (12 months) might be too short to reveal some of the evaluated long-term effects of the intervention (i.e. heart remodeling).

\section{Conclusions}

1. Impedance cardiography offers an effective option of individualized drug selection in $\mathrm{AH}$.

2. Some patients can benefit from the hemodynamically-guided pharmacotherapy in significantly greater BP reduction.

3. The effect of hemodynamically-guided pharmacotherapy persists over time. 
4. Hemodynamic effects of such treatment approach, especially those of prognostic value (central BP, myocardial hypertrophy), should be evaluated in further studies including patients with resistant hypertension, heart failure, diabetes mellitus and chronic kidney disease.

\section{Acknowledgments}

The study was supported by the Ministry of Science and Higher Education/Military Institute of Medicine, Warsaw, Poland (grants no 148/WIM and 335/WIM).

We would like to thank the medical staff of our department for nursing care and data collection.

\section{Conflict of interest: None declared}

\section{References}

1. Kearney PM, Whelton M, Reynolds K, Muntner P, Whelton PK, He J. Global burden of hypertension: Analysis of worldwide data. Lancet, 2005; 365: 217-223.

2. Mancia G, Fagard R, Narkiewicz K et al. 2013 ESH/ESC Guidelines for the management of arterial hypertension: The Task Force for the management of arterial hypertension of the European Society of Hypertension (ESH) and of the European Society of Cardiology (ESC). J Hypertens, 2013; 31: 1281-1357. doi: 10.3109/08037051.2014.868629.

3. James PA, Oparil S, Carter BL et al. 2014 evidence-based guideline for the management of high blood pressure in adults: Report from the panel members appointed to the Eighth Joint National Committee (JNC 8). JAMA, 2014; 311: 507-520. doi: 10.1001/ jama.2013.284427.

4. Ventura HO, Taler SJ, Strobeck JE. Hypertension as a hemodynamic disease: the role of impedance cardiography in diagnostic, prognostic, and therapeutic decision making. Am J Hypertens, 2005; 18: 26S-243S.

5. Taler SJ, Textor SC, Augustine JE. Resistant hypertension: Comparing hemodynamic management to specialist care. Hypertension, 2002; 39: 982-988.

6. Smith RD, Levy P, Ferrario CM. Consideration of Noninvasive Hemodynamic Monitoring to Target Reduction of Blood Pressure Levels Study Group: Value of Noninvasive Hemodynamics to Achieve Blood Pressure Control in Hypertensive Subjects (The CONTROL Trial). Hypertension, 2006; 47: 769-775.

7. Krzesiński P, Gielerak GG, Kowal JJ. A “patient-tailored” treatment of hypertension with use of impedance cardiography: A randomized, prospective and controlled trial. Med Sci Monit, 2013; 19: 242-250. doi: 10.12659/MSM.883870
8. Ferrario CM, Flack JM, Strobeck JE, Smits G, Peters C. Individualizing hypertension treatment with impedance cardiography: A meta-analysis of published trials. Ther Adv Cardiovasc Dis, 2010; 4: 5-16. doi: 10.1177/17539447709348236.

9. Trudeau L. Central blood pressure as an index of antihypertensive control: Determinants and potential value. Can J Cardiol, 2001; 30 (5 suppl.): S23-8. doi: 10.1016/j.cjca.2014.03.010.

10. Pauca AL, O'Rourke MF, Kon ND. Prospective evaluation of a method for estimating ascending aortic pressure from the radial artery pressure waveform. Hypertension, 2001; 38: 932-937.

11. Nagueh SF, Appleton CP, Gillebert TC, et al. Recommendations for the evaluation of left ventricular diastolic function by echocardiography. Eur J Echocardiogr, 2009; 10: 165-193. doi: 10.1093/ ejechocard/jep007.

12. Sramek BB, Tichy JA, Hojerova M, Cervenka V. Normohemodynamic goal-oriented antihypertensive therapy improves the outcome. Am J Hypertens, 1996; 9: 141A.

13. Flack JM. Noninvasive hemodynamic measurements: An important advance in individualizing drug therapies for hypertensive patients. Hypertension, 2006; 47: 646-647.

14. Palatini P. Heart rate as an independent risk factor for cardiovascular disease: Current evidence and basic mechanisms. Drugs, 2007; 67 (suppl. 2): 3-13.

15. Palatini P, Dorigatti F, Zaetta V et al. HARVEST Study Group. Heart rate as a predictor of development of sustained hypertension in subjects screened for stage 1 hypertension: the HARVEST Study. J Hypertens, 2006; 24: 1873-1880.

16. Williams B. Recent hypertension trials: implications and controversies. J Am Coll Cardiol, 2005; 45: 813-827.

17. Fadl Elmula FE, Hoffmann P, Larstorp AC et al. Adjusted drug treatment is superior to renal sympathetic denervation in patients with true treatment-resistant hypertension. Hypertension, 2014; 63: 991-999. doi: 10.1161/HYPERTENSIONAHA.114.03246.

18. Krzesinski P, Gielerak G, Stanczyk A, Piotrowicz K, Skrobowski A. 9C.03: the benefit from hemodynamically guided antihypertensive therapy depends on baseline blood pressure. J Hypertens, 2015; 33 (suppl. 1): e123-124. doi: 10.1097/01. hjh.0000467684.31931.63.

19. Fagard RH, Celis H, Thijs L, Wouters S. Regression of left ventricular mass by antihypertensive treatment: A meta-analysis of randomized comparative studies. Hypertension, 2009; 54: 1084-1091. doi: 10.1161/HYPERTENSIONAHA.109.136655.

20. Williams B, Lacy PS, Thom SM, Cruickshank K et al.; CAFE Investigators; Anglo-Scandinavian Cardiac Outcomes Trial Investigators; CAFE Steering Committee and Writing Committee. Differential impact of blood pressure-lowering drugs on central aortic pressure and clinical outcomes: Principal results of the Conduit Artery Function Evaluation (CAFE) study. Circulation, 2006; 113: 1213-1225.

21. Nieminen T, Kähönen M, Kööbi T. Letter by Nieminen et al. regarding article, "Differential impact of blood pressure-lowering drugs on central aortic pressure and clinical outcomes: principal results of the Conduit Artery Function Evaluation (CAFE) study". Circulation, 2006; 114: e536. 